\title{
Evaluation des Modellprojektes „Rezept für Bewegung“ in Baden-Württemberg mittels explorativer qualitativer Befragung
} Evaluation of the Model project "Prescription for Exercise" in Baden-Württemberg: An Explorative Qualitative Survey

\section{(ㄷ) (1) ()}

Autoren

Sigrid Emerich, Christine Preiser, Monika A Rieger

Institut

Institut für Arbeitsmedizin, Sozialmedizin und Versorgungsforschung, Universitätsklinikum Tübingen, Tübingen

\section{Schlüsselwörter}

Rezept für Bewegung, Gesundheitsförderung, Prävention, Qualitative Evaluation

Key words

prescription for exercise, health promotion, prevention, qualitative exploration

online publiziert $\quad 12.08 .2020$

\section{Bibliografie}

Gesundheitswesen 2021; 83: 24-32

DOI 10.1055/a-1075-2149

ISSN $0941-3790$

(C) 2020. The Author(s).

This is an open access article published by Thieme under the terms of the Creative Commons Attribution-NonDerivative-NonCommercial-License, permitting copying and reproduction so long as the original work is given appropriate credit. Contents may not be used for commecial purposes, or adapted, remixed, transformed or built upon. (https://creativecommons.org/ licenses/by-nc-nd/4.0/)

Georg Thieme Verlag KG, Rüdigerstraße 14,

70469 Stuttgart, Germany

Korrespondenzadresse

Christine Preiser

Institut für Arbeitsmedizin

Sozialmedizin und Versorgungsforschung

Universitätsklinikum Tübingen

Wilhelmstraße 27

72074 Tübingen

christine.preiser@med.uni-tuebingen.de

\section{ZUSAMMENFASSUNC}

Ziel der Studie Bewegung hat auf die Gesundheit einen positiven Effekt, der bereits in vielen Studien bewiesen wurde. Bei der Unterstützung der PatientInnen hin zu mehr Bewegung haben ÄrztInnen eine besondere Rolle. Vor diesem Hintergrund wurde 2009 das Modellprojekt „Rezept für Bewegung“ (RfB) in
Deutschland ins Leben gerufen. Hierbei können ÄrztInnen ihren PatientInnen ein tatsächliches Rezept für Bewegung ausstellen. In Baden-Württemberg wurde dieses Angebot von 2015 bis 2016 in 4 Modellregionen erprobt. Ziel der vorliegenden Studie war es, nach dem Ende der Erprobung die Verwendung des RfB durch ÄrztInnen in Baden-Württemberg zu evaluieren.

Methodik Die an dem Programm teilnehmenden ÄrztInnen wurden qualitativ befragt. Die Befragung wurde anhand eines strukturierten Interviewleitfadens telefonisch durchgeführt. Ergebnisse Die qualitative Befragung zeigte eine positive Einstellung der ÄrztInnen gegenüber dem RfB. Als hemmende Faktoren für die Ausstellung des RfB wurden fehlende örtliche Angebote und die fehlende direkte Kostenübernahme der Krankenkasse für die empfohlenen Bewegungsangebote angegeben. Weitere Einflussgrößen stellten die Eigenmotivation der PatientInnen sowie die antizipierte Wahrscheinlichkeit der Umsetzung der Bewegungsempfehlung dar.

Schlussfolgerung Durch die vorliegende qualitative Erhebung konnten nicht nur Hemmnisse bei der Ausstellung des RfB durch Ärztlnnen und der Umsetzung der Bewegungsempfehlungen durch die PatientInnen, sondern auch förderliche Faktoren mit Blick auf die künftige Verwendung des RfB außerhalb des Modellvorhabens dokumentiert werden.

\section{ABSTRACT}

Objective Exercise has a positive effect on health, which has already been proven in many studies. Doctors have an important role in helping patients to get more exercise. Against this background, the preventive model project "Exercise on Prescription" (RfB) was launched in Germany in 2009. This allows doctors to give their patients a prescription for exercise. In Baden-Württemberg, this offer was tested in 4 regions from 2015 to 2016 . The aim of the present study was to evaluate the use of the RfB by doctors in Baden-Württemberg at the end of the trial phase by means of qualitative interviews of physicians participating in the program in 2017.

Methods The telephone interviews were conducted using a structured interview guide.

Results The qualitative survey showed a positive attitude of the physicians towards the RfB. The lack of local offers and the 
lack of direct cost reimbursement by the statutory health insurances were cited as inhibiting factors for the issuance of the RfB. Further influencing factors were the self-motivation of the patients and the anticipated probability of implementation.
Conclusion The present qualitative survey not only documented the obstacles to RfB being issued by doctors and the patients' implementation of the physical activity recommendations, but also beneficial factors with regard to the future use of the RfB outside the model project.

\section{Einleitung}

Bewegung im Sinne „gesundheitsförderlicher körperlicher Aktivität“ [1] ist ein zentrales Element der Verhaltensprävention von chronischen und onkologischen Erkrankungen [2,3]. Es gibt eine Vielzahl von Maßnahmen, die die Motivation zu mehr Bewegung steigern und verbessern sollen. Als ein präventives Angebot wurde in Deutschland im Jahr 2009 zu diesem Zweck in mehreren Bundesländern das „Rezept für Bewegung“ (RfB) gemeinsam von der Deutschen Gesellschaft für Sportmedizin und Prävention (DGSP), dem Deutschen Olympischen Sportbund (DOSB) und der Bundesärztekammer (BÄK) ins Leben gerufen [4]. Hierbei handelt es sich um ein tatsächliches Rezept, auf dem Haus- und FachärztInnen ihren PatientInnen Aktivitäten in einem der 4 Trainingsschwerpunkte Herz-Kreislauf, Muskel-Skelett-System, Entspannung/Stressbewältigung und Koordination/motorische Fähigkeiten empfehlen können. Hierzu sollen Anbieter genutzt werden, die mit dem Qualitätssiegel SPORT PRO GESUNDHEIT zertifiziert wurden [5]. In Baden-Württemberg wurde das RfB von 2015 bis 2016 im Rahmen eines Modellprojektes der Landesärztekammer (LÄK) in den Regionen Biberach, Freiburg, Heilbronn und Karlsruhe eingeführt [6, 7]. Die Unterlagen für die Teilnahme am Modellprojekt wurden während der Laufzeit von mehr als 370 ÄrztInnen (> 22\% der Zielgruppe von ca. 1800 ÄrztInnen in den 4 Modell-Landkreisen) angefordert [8]. Nach dem zweiten Jahr der Erprobungsphase wurde durch die LÄK Baden-Württemberg zu Beginn des Jahres 2017 mittels eines Fragebogens eine kurzgefasste standardisierte Befragung der ÄrztInnen durchgeführt, die die Unterlagen angefordert hatten. Dieser Fragebogen enthielt u. a. 5 Fragen zur Erfassung der Einstellung zu Bewegungsempfehlung und zur Nutzung des RfB, ohne dass diese Aspekte vertieft erhoben wurden. Vor diesem Hintergrund bot das Institut der LÄK im Vorfeld an, er- gänzend mittels qualitativer Interviews vertiefende Informationen bei den Ärztlnnen zu erheben.

Ziel der vorliegenden wissenschaftlichen Untersuchung war es, die standardisierten Befragungsergebnisse durch Informationen zur Verwendung und Akzeptanz des RfB sowie zu hinderlichen und förderlichen Faktoren der Verwendung zu ergänzen. Dabei standen folgende Fragen im Mittelpunkt:

1) Mit welcher Haltung wird das RfB von den ÄrztInnen ausgestellt?

2) Wie wirken sich u. a. folgende Aspekte auf die Motivation der ÄrztInnen zur Bewegungsberatung aus: ausreichende Verfügbarkeit von Sportangeboten, Kostenübernahme durch gesetzliche Krankenkassen, Rückmeldungen von PatientInnen über Effekte von Bewegung, spezifische (z.B. arbeitsbedingte) Anforderungen und angenommene Wirksamkeit von Bewegungsangeboten?

3) Gibt es weitere hemmende Gründe für die Ausstellung des RfB, die in dem Fragebogen nicht erfragt wurden?

\section{Methodik}

Im Sinne eines Vertiefungsdesigns [9] wurden von August bis September 2017 die im Kurzfragebogen erhobenen Erfahrungen und Haltungen der Haus- und FachärztInnen zum RfB mittels qualitativer Verfahren der empirischen Sozialforschung vertiefend analysiert.

Die erste Information über die qualitative Evaluation (Thema und Länge des Interviews) erfolgte bereits durch die LÄK BadenWürttemberg im Rahmen der standardisierten Befragung, verbunden mit der Bitte um Teilnahme. Auf 35 der 114 an die LÄK zurückgesandten Fragebögen erklärten sich ÄrztInnen zu einem Interview bereit. Nach dem Prinzip der maximalen strukturellen Variation

> Tab. 1 Sampling-Kriterien - Zusammensetzung der Stichprobe.

\begin{tabular}{|c|c|c|}
\hline $\begin{array}{l}\text { Sampling-Kriterien (Basis: Angaben im Fragebogen } \\
\text { der Landesärztekammer Baden-Württemberg) }\end{array}$ & Geplantes Sample & Tatsächliches Sample \\
\hline $\begin{array}{l}\text { Selbstbild: Antwort auf die Aussage „Beratung zur } \\
\text { Förderung der körperlichen Aktivität ist eine ärztliche } \\
\text { Aufgabe“ (gruppiert zu „ja“ oder „nein“)* }\end{array}$ & Ja: $7 \mathrm{HÄ}^{*}{ }^{*}, 4 \mathrm{FÄ}$ & Ja: 5 HÄ, 3 FÄ \\
\hline $\begin{array}{l}\text { Häufigkeit der Ausstellung des Rezept für } \\
\text { Bewegung }{ }^{* * *}\end{array}$ & $\begin{array}{l}\text { - Häufig: } 3 \text { HÄ, } 2 \text { FÄ } \\
\text { - Weniger häufig: } 2 \text { HÄ, } 1 \text { FA } \\
\text { - Nie: } 2 \text { HÄ, } 1 \text { FA }\end{array}$ & $\begin{array}{l}\text { - Häufig: } 1 \text { HA, } 2 \text { FÄ } \\
\text { - Weniger häufig: } 2 \text { HÄ, } 0 \text { FA } \\
\text { - Nie: } 2 \text { HÄ, } 1 \text { FA }\end{array}$ \\
\hline $\begin{array}{l}\text { Hemmende Gründe } * * * * \\
\text { - Innere Faktoren: fehlende Vergütung, Zeitmangel, } \\
\text { andere Präventionsfelder sind wichtiger } \\
\text { - Äußere Faktoren: zu wenig Angebot, fehlende } \\
\text { Leistungserstattung für Patientlnnen } \\
\text { - Patientenbezogene Faktoren: PatientInnen zu alt, } \\
\text { mangelndes Interesse der PatientInnen an Bewegung }\end{array}$ & $\begin{array}{l}\text { - Innere Faktoren: } 2 \text { HÄ, } 1 \text { FA } \\
\text { - Äußere Faktoren: } 3 \text { HÄ, } 2 \text { FÄ } \\
\text { - Patientenbezogene Faktoren: } 2 \text { HÄ, } 1 \text { FA } \\
\text { - Keine Angaben gemacht: } 2 \text { HÄ, } 1 \text { FA }\end{array}$ & $\begin{array}{l}\text { - Innere Faktoren: } 2 \text { HÄ, } 2 \text { FÄ } \\
\text { - Äußere Faktoren: } 2 \text { HÄ, } 2 \text { FÄ } \\
\text { - Patientenbezogene Faktoren: } 3 \text { HÄ, } 1 \text { FA } \\
\text { - Keine Angaben gemacht: } 1 \text { HA, } 1 \text { FA }\end{array}$ \\
\hline \multicolumn{3}{|c|}{$\begin{array}{l}{ }^{*} \text { In allen uns vorliegenden } 114 \text { Fragebögen lautete die Antwort auf diese Frage „ja“ oder „eher ja“. }{ }^{*} * \text { (HA }=\text { Hausarzt/ Hausärztin; FA = Facharzt/ } \\
\text { Fachärztin). } *^{*} \text { Antwortkategorien im Fragebogen (ohne Spezifikation). }{ }^{*} * * \text { Mehrfachnennungen möglich, Gruppierung der Fragebogen-Items } \\
\text { durch die Autorinnen }\end{array}$} \\
\hline
\end{tabular}




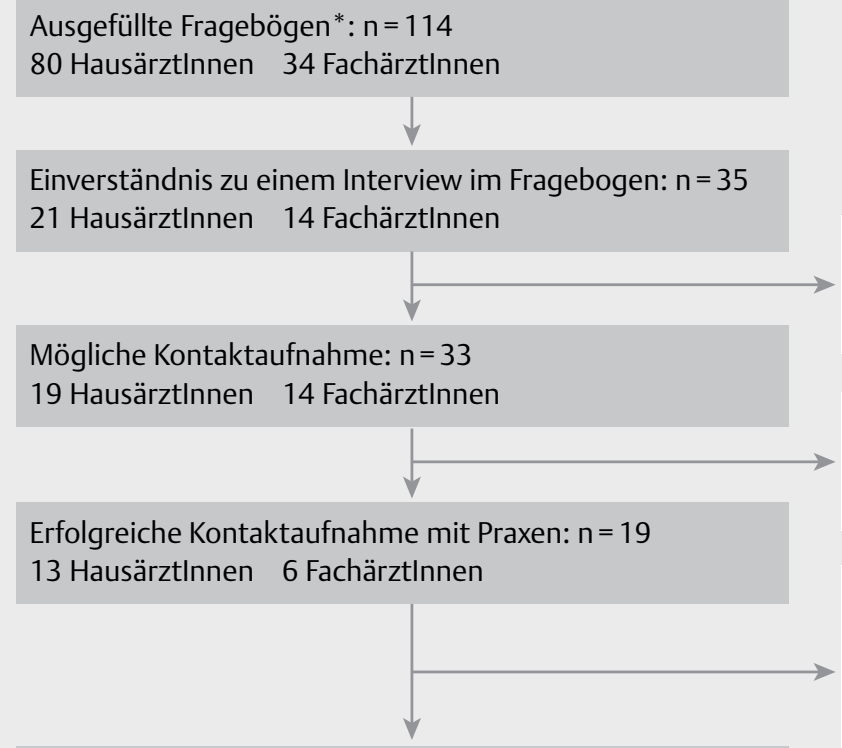

Rekrutierungsgespräch mit ÄrztInnen: $\mathrm{n}=11$

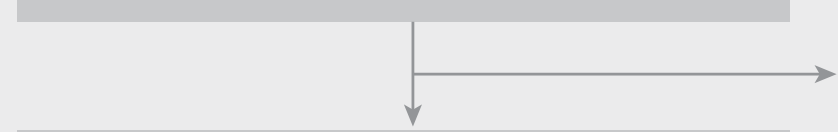

Durchgeführte Interviews: $\mathrm{n}=9$

6 HausärztInnen 3 FachärztInnen

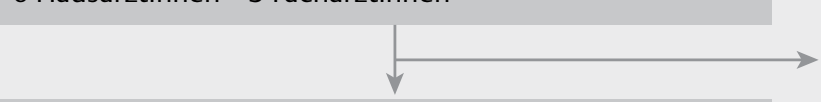

Ausgewertete Interviews: $\mathrm{n}=8$

5 HausärztInnen 3 FachärztInnen
Nicht eingeschlossene Ärztlnnen: $\mathrm{n}=2$

2 HausärztInnen 0 Fachärztlnnen

(1× schriftlicher Wiederspruch, $1 \times$ nicht mehr in Praxis tätig)

Nicht eingeschlossene ÄrztInnen: $n=14$

6 HausärztInnen 8 FachärztInnen

(kein persönlicher Kontakt mit Praxis hergestellt)

Nicht eingeschlossene ÄrztInnen: $\mathrm{n}=8$

6 Hausärztlnnen 2 FachärztInnen

(1× fehlende Zuordenbarkeit in Gemeinschaftspraxis,

$2 \times$ Rückzug der Einwilligung, $1 \times$ Kinderärztln, $4 \times$ kein erneuter Kontakt mit ÄrztInnen hergestellt)

Ausgeschlossene Ärztlnnen/Drop out: $\mathrm{n}=2$

1 Hausärztln 1 Fachärztln

(1× Rückzug der Einwilligung, 1× keine Terminfindung in

Erhebungszeitraum)

Ausgeschlossene Ärztln: $n=1$

1 Hausärztln 0 Fachärztln

(1× Kinderärztin)

- Abb. 1 Sampling-Verlauf * exakte Anzahl der versendeten Fragebögen ist den Autorinnen nicht bekannt (ca. 370).

[10] wurde ein möglichst kontrastreiches Sample angestrebt, um die Heterogenität des Untersuchungsfeldes abzubilden. Forschungsrelevante Kriterien waren folgende Angaben aus der standardisierten Befragung: Haus- oder Fachärztln, Selbstbild in Bezug auf die Beratung zu körperlicher Aktivität, berichtete Häufigkeit der Ausstellung des RfB und Antwortverhalten bei den erfragten hemmenden Gründen ( $\triangleright$ Tab. 1).

Die ausgewählten ÄrztInnen wurden vom Projektteam des Instituts telefonisch kontaktiert und erneut über die Evaluation informiert. Dabei erwies es sich als nicht immer einfach, die angedachten InterviewpartnerInnen zu erreichen: Besonders bei Gemeinschaftspraxen war eine Bestimmung der Person schwierig, wenn im Fragebogen keine Kontaktperson angegeben worden war. Auch waren viele der angedachten InterviewpartnerInnen zum Zeitpunkt der versuchten Kontaktaufnahme für mehrere Wochen im Urlaub. Hinzu kam, dass teilweise Praxen zwar erreicht werden konnten, aber ein Kontakt nicht über die Medizinischen Fachangestellten hinaus zustande kam. Aufgrund der kurzen Projektlaufzeit und der begrenzten Anzahl der ÄrztInnen, die zu einem Interview eingewilligt hatten, wurde von der geplanten strengen Sampling-Auswahl abgewichen und mit allen telefonisch erreichbaren und zustimmenden ÄrztInnen (9 Personen) ein Interview geführt. Davon wurde aus methodischen Gründen das Interview mit einem Pädiater nicht in die Datenanalyse eingeschlossen, da dieser schilderte, dass er nicht die PatientInnen selbst, sondern deren Eltern berate. In \ Abb. 1 sind der Rekrutierungsverlauf und die Gründe für einen nicht möglichen Einschluss von ÄrztInnen dargestellt. Die geplante und die tatsächliche Zusammensetzung der InterviewpartnerInnen sind in > Tab. 1 wiedergegeben. Die Telefoninterviews wurden mit Hilfe eines vom Institut entwickelten Leitfadens geführt und dauerten durchschnittlich 10 Minuten.

Zum Zwecke der Qualitätssicherung wurden die durch einen kommerziellen Dienstleister transkribierten Daten von 2 Personen (SE, CP) zunächst getrennt voneinander ausgewertet und dann gemeinsam diskutiert, um die intersubjektive Übereinstimmung zu erhöhen [11,12]. Die Daten wurden mittels qualitativer Inhaltsanalyse ausgewertet [13]. In deren Mittelpunkt stehen die Erarbeitung eines Kategoriensystems und die Abbildung aller relevanten Textstellen in mindestens einer Kategorie des Kategoriensystems. Die Erstellung der Kategorien erfolgte gemischt induktiv-deduktiv: Mögliche Kategorien wurden deduktiv aus den Fragen des Leitfadens abgeleitet und weitere Kategorien induktiv aus dem Material erschlossen. Induktive und deduktive Kategorien wurden anschließend in eine hierarchische Ordnung (Hauptkategorien Unterkategorien) gebracht. Das Kategoriensystem wurde erneut am 
Material getestet und dann auf die verbleibenden Interviews angewendet. Die nachfolgenden Ergebnisse wurden Ende 2017 kurzgefasst in einem unveröffentlichten Abschlussbericht zur Vorlage bei der Landesärztekammer Baden-Württemberg dargestellt [14].

\section{Ergebnisse}

Das Kategoriensystem enthielt 3 Hauptthemen: die Ausstellung des RfB, die Umsetzung der Bewegungsempfehlung und Verbes- serungsvorschläge. Im Text wird jeweils auf die nummerierten Ankerbeispiele in > Tab. 2 verwiesen.

\section{Die Ausstellung des Rezeptes}

\section{Gründe für die Ausstellung}

Das Konzept des RfB wird von den Befragten einheitlich positiv bewertet (1.1). Die ÄrztInnen stellen das RfB aus verschiedenen Gründen aus. Die Empfehlung zu mehr Bewegung werde im Vergleich zur rein mündlichen Empfehlung aufgewertet (1.2). Das RfB sei eine gute Alternative zur Verschreibung von Medikamenten und stelle

- Tab. 2 Kategoriensystem mit Ankerbeispielen.

\begin{tabular}{|c|c|c|}
\hline \multicolumn{3}{|r|}{ 1. Das Rezept ausstellen } \\
\hline \multicolumn{3}{|r|}{ Gründe } \\
\hline 1.1 & RfB wird positiv bewertet & „a, ich finde es gut, dass man das nun eingerichtet hat oder beschlossen hat. Rezept für Bewegung, ja“. (FA 3) * \\
\hline 1.2 & $\begin{array}{l}\text { Aufwertung von } \\
\text { Bewegungsempfehlung }\end{array}$ & $\begin{array}{l}\text { „Ja ich meine die Realität ... ja also, ich finde es schon mal wichtig, dass man nicht nur sagt, sie sollen sich mehr } \\
\text { bewegen, sondern dass man dann auch sagt in Form eines Rezeptes, man gibt dem ... der Bewegung ein gewisses } \\
\text { Mehr an Wertigkeit, was ja auch dann [....“.. (FA 2) }\end{array}$ \\
\hline 1.3 & $\begin{array}{l}\text { Alternative zu Medika- } \\
\text { menten }\end{array}$ & $\begin{array}{l}\text { "Ja das ist eine prima Sache, dass wir die Leute nicht nur mit Medikamenten abfüttern, sondern ihnen auch mal was } \\
\text { anderes anreichen oder anraten“. (HA 5) }{ }^{*}\end{array}$ \\
\hline 1.4 & Fixierung einer Absprache & $\begin{array}{l}\text { „Es ist ein Versuch, durch eine schriftliche Form und eine Fixierung, eine Absprache oder eine Verhaltensänderung } \\
\text { beim Patienten zu befördern“. (HA 3) }\end{array}$ \\
\hline 1.5 & $\begin{array}{l}\text { Verbesserung der } \\
\text { Nachhaltigkeit }\end{array}$ & $\begin{array}{l}\text { „[...] damit es nicht versandet, ist es wie bei anderen Rezepten auch, es ist natürlich gut, wenn so was noch mal der } \\
\text { Patient schriftlich hat und zu Hause die Motivation vielleicht noch mal mehr hat, als er es vielleicht so hätte“. (HA 1) }\end{array}$ \\
\hline 1.6 & $\begin{array}{l}\text { Auslagerung der } \\
\text { Aufklärungsarbeit }\end{array}$ & $\begin{array}{l}\text { „[...] wenn da ein beschleunigter Takt besteht bei den Patienten und ich habe jetzt zu wenig Zeit, um dem } \\
\text { Patienten das ausführlich selbst zu erläutern, was er machen und tun sollte und dass man dann noch was in die } \\
\text { Hand drückt und sagt man: Das ist ein ernst gemeintes Rezept und ich möchte haben, dass Sie das umsetzen und } \\
\text { ich möchte Sie in vier Wochen wiedersehen, um darüber zu reden. Das könnte ich mir nach wie vor auch trotzdem } \\
\text { als gutes Konzept vorstellen“. (HA 1) }\end{array}$ \\
\hline \multicolumn{3}{|r|}{ Hinderungsgründe } \\
\hline 1.7 & Fehlende Vergütung & $\begin{array}{l}\text { „[...] prinzipiell würde man sich natürlich wünschen, dass hinter einer Verordnung eine Erstattung steht, in welcher } \\
\text { Form auch immer, [...]“. (FA 2) }\end{array}$ \\
\hline 1.8 & $\begin{array}{l}\text { Bewegungsempfehlung } \\
\text { in der alläglichen Arbeit } \\
\text { bereits vorhanden }\end{array}$ & $\begin{array}{l}\text { „Im Rahmen eines Gesamtkonzeptes, wie es meine Alltagspraxis ist, wo Sportmedizin sowieso eine große Rolle } \\
\text { spielt, ist das ein solches Thema, dass es so allgegenwärtig ist, dass ich es persönlich dann eben nicht brauche oder } \\
\text { nicht benutze“. (HA 1) }\end{array}$ \\
\hline 1.9 & $\begin{array}{l}\text { Einschätzung der eigenen } \\
\text { Fähigkeiten }\end{array}$ & $\begin{array}{l}\text { „[...] das ist auch die Frage unserer Motivationskunst, das doch zu machen. Also wie gesagt, das sind da ... das läuft } \\
\text { manchmal auch ganz witzig ab, wie man zu solchen Motivationen kommt und dann brauche ich dann eben nicht } \\
\text { noch mal das Rezept am Schluss [...]“. (HA 1) }\end{array}$ \\
\hline 1.10 & $\begin{array}{l}\text { Rezept ist nicht } \\
\text { nachdrücklich genug }\end{array}$ & $\begin{array}{l}\text { „Deswegen ist das eigentlich leider wider den Verordnungsgedanken eine Empfehlung. Man liest beim Raucher auch } \\
\text { nicht mehr: Schränken Sie Ihren Rauchkonsum ein. Anstatt - was weiß ich -: Verordnen Sie Frischluft“. (FA 2) }\end{array}$ \\
\hline 1.11 & $\begin{array}{l}\text { Macht im privatärztlichen } \\
\text { Setting keinen Sinn }\end{array}$ & „In meinem privatärztlichen Bereich, wo viel Zeit für die Patienten da ist, brauche ich es einfach nicht“. (HA 1) \\
\hline 1.12 & $\begin{array}{l}\text { Empfindlichkeiten der } \\
\text { PatientInnen }\end{array}$ & $\begin{array}{l}\text { „Es gibt auch die Leute, die sehr empfindlich sind, wenn man sie wiederholt auf Defizite oder Verbesserungsmög- } \\
\text { lichkeiten anspricht, und da ist es dann ganz ehrlich gesagt so, wenn ich das zweimal gemacht habe, und die Leute } \\
\text { da sehr empfindlich sind und deutliche Anzeichen machen, dass sie da nicht mehr darauf angesprochen werden } \\
\text { wollen, dann halte ich mich die nächsten Male auch eher zurück“. (HA 4) }\end{array}$ \\
\hline 1.13 & $\begin{array}{l}\text { Einschätzung der } \\
\text { Adhärenz }\end{array}$ & $\begin{array}{l}\text { „Wenn man so über die Jahre schaut, kann man manchmal kleine Erfolgserlebnisse haben. Aber häufig ist es so, } \\
\text { dass es ja doch eher frustrierend ist. Also Wunder verspreche ich mir jetzt da nicht davon“. (HA 3) }\end{array}$ \\
\hline 1.14 & Eigene Frustration & $\begin{array}{l}\text { "Ja leider ist das sehr frustran. Also man muss sich selbst immer motivieren, damit... das nicht aufzugeben. } \\
\text { Manchmal hat man Erfolgserlebnisse. Aber ich sage mal, wenn bei } 10 \text { Patienten das } 1 \text { oder } 2 \text { sind, dann ist das viel“. } \\
\text { (HA 3) }\end{array}$ \\
\hline 1.15 & $\begin{array}{l}\text { Keine geeigneten } \\
\text { Strukturen vor Ort }\end{array}$ & $\begin{array}{l}\text { „Ich habe mich dann halt auch erkundigt, wer das überhaupt macht, welcher Sportverein und wer da zuständig ist, } \\
\text { wenn man das ausstellen würde, und dann hat sich eben bei mir am Ort herausgestellt, dass da zwar eine } \\
\text { Übungsleiterin dafür auf dem Papier stand, dass das [SPORT FÜR GESUNDHEIT-Angebot] aber überhaupt noch nicht } \\
\text { umgesetzt wurde oder begonnen wurde“. (FA 3) }\end{array}$ \\
\hline 1.16 & $\begin{array}{l}\text { Fehlende Kenntnisse zu } \\
\text { Abläufen }\end{array}$ & $\begin{array}{l}\text { „Also dieser Weg ist bei mir irgendwie noch überhaupt nicht drin, dass man das jetzt wirklich auf Rezept ... und ich } \\
\text { weiß einfach auch tatsächlich nicht, wie genau die praktischen Abläufe danach sind. Deshalb ist das glaube ich } \\
\text { noch nicht wirklich hier eingeschlagen“. (HA 5) }\end{array}$ \\
\hline 1.17 & $\begin{array}{l}\text { Geht zwischen anderen } \\
\text { Angeboten unter }\end{array}$ & $\begin{array}{l}\text { „Es ist nur, als dann im Alltag, wenn es immer mehr Möglichkeiten und Angebote gibt, ist es schwierig, da den } \\
\text { Überblick zu behalten“. (HA 4) }\end{array}$ \\
\hline
\end{tabular}


- Tab. 2 Fortsetzung.

\begin{tabular}{|c|c|c|}
\hline \multicolumn{3}{|r|}{ 2. Bewegungsempfehlung umsetzen } \\
\hline \multicolumn{3}{|r|}{ Motivation } \\
\hline 2.1 & $\begin{array}{l}\text { Niedrigschwelligeres } \\
\text { Wording }\end{array}$ & $\begin{array}{l}\text { „Also ich schlage den Leuten immer vor, dass sie das nicht Sport nennen, sondern Bewegung, weil Sport ja so eine } \\
\text { hohe Hürde hat“. (HA 5) }\end{array}$ \\
\hline 2.2 & $\begin{array}{l}\text { Integration von } \\
\text { Bewegung in Alltag }\end{array}$ & $\begin{array}{l}\text { „Und dass sie mit ganz kleinen Sachen anfangen, nämlich das, die Bewegung in den Alltag zu integrieren. Dass man } \\
\text { den Aufzug stehen lässt und die Treppe läuft“. (HA 5) }\end{array}$ \\
\hline 2.3 & $\begin{array}{l}\text { Integration von } \\
\text { Bewegung in Freizeit }\end{array}$ & $\begin{array}{l}\text { "Also so versuche ich die Leute zu kriegen oder auch jemand der Kinder hat, dass man sagt, gehen Sie mit Ihren } \\
\text { Kindern ins Schwimmbad und lassen die dann, wenn die groß genug sind, halt auch mal im Nichtschwimmerbecken } \\
\text { alleine und gehen dann mal ein paar Bahnen schwimmen, dass Sie sich ein bisschen auspowern können“. (HA 5) }\end{array}$ \\
\hline 2.4 & Ärztin/Arzt als Vorbild & $\begin{array}{l}\text { „[...] also das ist, würde ich sagen, dadurch, dass ich selber das auch vorlebe und nicht im übertriebenen Maße, } \\
{[\ldots . . . \text { ( (HA 1) }}\end{array}$ \\
\hline 2.5 & $\begin{array}{l}\text { Soziale Kontrolle durch } \\
\text { Trainingspartner }\end{array}$ & $\begin{array}{l}\text { „Aber sich am besten mit einem Freund oder mit dem Partner oder sowas zusammen für irgendwelche Bewegungs- } \\
\text { sachen aufzuraffen [...]. Wenn da andere Leute beteiligt sind, mit denen ich mich vielleicht auch gerne treffe, dann } \\
\text { ist es leichter mit der Motivation“. (HA 5) }\end{array}$ \\
\hline 2.6 & Prägendes Ereignis & $\begin{array}{l}\text { „[...] also die Motivation des Arztes allein ist es nicht. Es muss meist ein prägendes Ereignis sein: neue Diagnose von } \\
\text { dem Diabetes, ein Ereignis bei einem Verwandten oder nahestehendem Bekannten, Nachbarn oder Ähnlichem“. } \\
\text { (HA 2) }\end{array}$ \\
\hline 2.7 & Partnerschaft & $\begin{array}{l}\text { „Also am besten ist es, wenn einer sich in eine Person verliebt, die selber Sportler ist, dann ist er bereit, manches } \\
\text { mitzumachen, aber das ist auch der stärkste Motivator“. (HA 2) }\end{array}$ \\
\hline 2.8 & Finanzielle Anreize & $\begin{array}{l}\text { „Die sind da [Rehasport] ganz gut aufgehoben. In diesem Funktionstraining im Thermalbad. Also da sehen die } \\
\text { Leute auch für sich den Vorteil, einfach wirklich, das Thermalbad ist schon furchtbar teuer und da zahlen sie dann } 2 \\
\text { Euro Eintritt, also das ist eine große Motivation für viele“. (HA 5) }\end{array}$ \\
\hline 2.9 & Berufliche Zufriedenheit & $\begin{array}{l}\text { "Also ich denke schon, dass Leute, die weniger berufliche Belastung haben, das eher machen und vielleicht auch } \\
\text { Leute, die eine höhere Berufszufriedenheit haben, sich vielleicht auch leichter aufraffen können zu sowas“. (HA 5) }\end{array}$ \\
\hline \multicolumn{3}{|r|}{ Hemmnisse } \\
\hline 2.10 & $\begin{array}{l}\text { Fehlende finanzielle } \\
\text { Unterstützung durch KK }\end{array}$ & $\begin{array}{l}\text { „[...] nimmt man es jetzt für den Bereich Adipositas oder chronischer Rücken oder wie auch immer, es gibt ja viele } \\
\text { Bereiche, machen sie (die KK) es (finanzielle Unterstützung) halt nicht und das ist extrem störend“. (FA 2) }\end{array}$ \\
\hline 2.11 & Lange Anfahrtswege & $\begin{array}{l}\text { „Ist halt auch immer das Thema mit Anfahrt usw., weil unser Einzugsgebiet doch größer ist und überregional. Und } \\
\text { dann ist eine Stunde Sport dann mit eineinhalb Stunden Fahrtzeit mit Verbindung in [Stadt] und dann sind es gleich } \\
\text { zweieinhalb Stunden Zeit, das ist dann der ganze Nachmittag an dem Tag. Da machen wenige Leute mit, muss man } \\
\text { sagen“. (FA 1) }\end{array}$ \\
\hline 2.12 & Compliance & „Also ich glaube, die Compliance ist nicht sehr hoch“. (FA 2) \\
\hline 2.13 & Bequemlichkeit & $\begin{array}{l}\text { „Die Patienten, die möchten gerne überall abgeholt werden. Also wenn ich jemanden hätte, der sagt, ich hol deine } \\
\text { Patienten bei dir an der Praxis ab, [...] könnte ich mir vorstellen, dass das etwas anderes ist, wie wenn sie sich auch } \\
\text { nur drei Häuser weiter an der Sporthalle treffen sollten, da ist schon wieder die erste, nun ja, Hemmung“. (HA 2) }\end{array}$ \\
\hline 2.14 & $\begin{array}{l}\text { Fehlende intrinsische } \\
\text { Motivation }\end{array}$ & $\begin{array}{l}\text { „Die Menschen wissen meistens, dass es sinnvoll wäre, wenn sie es täten, aber es gibt, nun ja, innere Motivation } \\
\text { (.....) die, die es immer wieder verhindern. Also wenn keine intrinsische Aktivität da ist, dann funktioniert es auch } \\
\text { auf noch so gutes Zureden nicht“. (HA 2) }\end{array}$ \\
\hline 2.15 & Scham & $\begin{array}{l}\text { „Ein Nichtsportler schämt sich, der kann sich überhaupt nicht vorstellen, dass andere Nichtsportler in der gleichen } \\
\text { Situation sind, und sie dann sozusagen zu dritt oder zu viert [...] auf einem gemeinsamen Leistungslevel beginnen } \\
\text { können und dann da eine Trainingssteigerung erfahren“. (HA 2) }\end{array}$ \\
\hline 2.16 & Umfeld bremst aus & $\begin{array}{l}\text { „Ob die dann wieder von der Partnerin gebremst werden, die sagen: Wieso stehst denn du schon auf, du musst } \\
\text { doch noch gar nicht?“ (HA 2) }\end{array}$ \\
\hline 2.17 & $\begin{array}{l}\text { Hohe berufliche } \\
\text { Belastung }\end{array}$ & $\begin{array}{l}\text { „Dass der dringend Bewegung braucht ist klar, ist ihm auch klar. Aber der sagt, er arbeitet jeden Tag zwölf Stunden. } \\
\text { Und dass der danach nicht mehr noch irgendwie was sportlich unternehmen mag, das kann man ihm nicht } \\
\text { verdenken, eigentlich“. (HA 5) }\end{array}$ \\
\hline \multicolumn{3}{|r|}{ Nachhaltigkeit } \\
\hline 2.18 & Abflachen der Motivation & $\begin{array}{l}\text { „Es wird mir versprochen, dass es getan wird, dass sie es anfangs getan haben und dann schläft es sehr häufig } \\
\text { wieder ein“. (HA 2) }\end{array}$ \\
\hline 2.19 & $\begin{array}{l}\text { Aktivierung von } \\
\text { Vorhandenem }\end{array}$ & $\begin{array}{l}\text { „[...] ich frage nach, ob sie früher schon einmal Sportler waren, weil einen, der schon Sportler war, ist leichter zu } \\
\text { reaktiveren als ein Couch-Potato, der noch nie Sport gemacht hat“. (HA 2) }\end{array}$ \\
\hline 2.20 & Fehlende Überwachung & „Dadurch, dass das natürlich nirgendwo überwacht wird, ist es immer schlecht“. (FA 2) \\
\hline 2.21 & $\begin{array}{l}\text { Steigert die Nachhaltig- } \\
\text { keit anderer Maßnahmen }\end{array}$ & $\begin{array}{l}\text { „Weil dieser Rehasport läuft ja eigentlich immer nur... also praktisch eine Verordnung und dann sagt man, sollten } \\
\text { die Leute ja auch fit genug sein, um das selbst zu machen und ich werde jetzt nach diesem Interview noch einmal } \\
\text { gucken, ob ich das dann vielleicht einsetze, um die weiterhin bei der Stange zu halten“. (HA 4) }\end{array}$ \\
\hline 2.22 & $\begin{array}{l}\text { Rückfall in erlernte } \\
\text { Verhaltensmuster }\end{array}$ & $\begin{array}{l}\text { „[...] das sind einfach alles ganz viele langjährig erlernte Verhalten, und in die fallen sie fast immer wieder zurück“. } \\
\text { (HA 2) }\end{array}$ \\
\hline
\end{tabular}


- Tab. 2 Fortsetzung

\begin{tabular}{|l|l|l|}
\hline \multicolumn{2}{|c|}{ 3. Verbesserungsvorschläge } \\
\hline 3.1 & $\begin{array}{l}\text { Finanzielle Unterstützung } \\
\text { durch Kasse für } \\
\text { PatientInnen }\end{array}$ & $\begin{array}{l}\text { „Ich würde mir wünschen, dass wenn man so Bewegung verordnet, auch die Kasse mitmacht in irgendeiner Art und } \\
\text { Weise, die Bewegung finanziell zu unterstützen. Bei jedem Medikament machen sie es auch [...]“. (FA 2) }\end{array}$ \\
\hline 3.2 & $\begin{array}{l}\text { Finanzielle Unterstützung } \\
\text { durch Kasse für ÄrztInnen }\end{array}$ & $\begin{array}{l}\text { "Also ich sage mal so, prinzipiell würde man sich natürlich wünschen, dass hinter einer Verordnung eine Erstattung } \\
\text { steht, in welcher Form auch immer“. (FA 2) }\end{array}$ \\
\hline 3.3 & $\begin{array}{l}\text { Mediale Aufmerksamkeit } \\
\text { steigern }\end{array}$ & $\begin{array}{l}\text { "Möglicherweise in weiteren Medien bewerben und, wenn es natürlich der Orthopäde genauso erwähnt wie ich } \\
\text { und der Kardiologe auch so wie ich und der Diabetologe auch so wie ich [...], dann wünschte ich mir, dass das } \\
\text { offters Anklang findet“. (HA 2) }\end{array}$ \\
\hline${ }^{*}$ (HA = Hausarzt/ Hausärztin; FA = Facharzt/ Fachärztin) \\
\hline
\end{tabular}

eine Erweiterung in den Handlungsmöglichkeiten der ÄrztInnen dar (1.3). Zusätzlich fixiere die schriftliche Form die Absprache, was sich förderlich auf eine Verhaltensänderung bei PatientInnen auswirken könne (1.4). Daneben werden eine verbesserte Motivation der PatientInnen und damit eine größere Nachhaltigkeit der Bewegungsempfehlung genannt (1.5). Das Rezept gebe ÄrztInnen darüber hinaus die Möglichkeit, trotz Zeitdruck in ihrem Arbeitsalltag den PatientInnen beratend und unterstützend zur Seite zu stehen und sie zur weiteren Beratung an bestehende Angebote zu überweisen (1.6).

\section{Hinderungsgründe für die Ausstellung}

Trotz der positiven Bewertung des RfB wurden von den Befragten vielfältige Gründe dafür genannt, warum sie das Rezept eher nicht ausstellen. Als einen Hinderungsgrund benennen sie die fehlende direkte Kostenübernahme seitens der gesetzlichen Krankenkassen und beziehen dies sowohl auf ihre eigene Arbeit als auch auf die PatientInnen (1.7). Befragte bezeichnen die Bewegungsempfehlung als einen alltäglichen Aspekt ihrer Arbeit und sprächen diese schon seit vielen Jahren in anderer Form aus (1.8). Deshalb sei für sie kein Mehrwert durch das RfB erkennbar. Gespräche und die eigene Fähigkeit, PatientInnen zu Bewegung zu motivieren, werden im Vergleich zum RfB als ein viel gewichtigeres Element bei der Motivation der PatientInnen gesehen (1.9). Die schriftliche Verordnung wird darüber hinaus als nicht nachdrücklich genug empfunden (1.10). Im privatärztlichen Bereich findet das Rezept laut einem Befragten keine Anwendung, da hier ausreichend Zeit für eben jene Gespräche gegeben sei (1.11). Die Befürchtung einer negativen Reaktion der PatientInnen auf die Aufforderung zu mehr Bewegung hält Befragte davon ab, das RfB auszustellen, da sie die Beziehung zu den PatientInnen nicht beeinträchtigen wollen (1.12). Hinzu kommt die Einschätzung einer zu geringen Adhärenz der PatientInnen im Hinblick auf die Bewegungsempfehlung. So erzählen ÄrztInnen von ihrem jahrelangen Versuch, PatientInnen zu mehr Bewegung zu motivieren, und dabei nur eine geringe Erfolgsquote zu haben (1.13). Dies führe bei den ÄrztInnen zu einer Frustration, welche sich negativ auf die häufige Ausstellung des RfBs auswirke, da sie keine Verbesserung der „Compliance“ durch das RfB annehmen (1.14). Als weiterer Hinderungsgrund wird genannt, dass vor Ort noch keine zertifizierten Angebote vorhanden seien (1.15). Hinzu kämen seitens der ÄrztInnen fehlende Kenntnisse über den Ablauf der Ausstellung und Umsetzung (1.16). Das rühre mitunter daher, dass das RfB in der Fülle der Möglichkeiten untergehe (1.17).

\section{Die Umsetzung der Bewegungsempfehlung}

Förderliche Faktoren für die Umsetzung der Bewegungsempfehlung durch die PatientInnen

Ärztlnnen nennen verschiedene Aspekte, die sich förderlich auf die tatsächliche Umsetzung der auf dem RfB gegebenen Empfehlung seitens der PatientInnen auswirken können. Niedrigschwelligkeit spiele dabei eine wichtige Rolle, um die Hürde der Umsetzung der Bewegungsempfehlung zu senken, indem die Bewegungsempfehlung nicht als Sport, sondern als Bewegung bezeichnet werde (2.1). Befragte raten ihren PatientInnen des Weiteren, die Bewegung in den Alltag (2.2) und die Freizeit zu integrieren (2.3). Als förderlich erachten Befragte auch die Motivation durch Dritte. So sehen sie sich selbst in einer Vorbildfunktion (2.4). Auch die soziale Kontrolle durch TrainingspartnerInnen kann laut Befragten zum Anfangen und Weitermachen motivieren (2.5). Besonders wirksame Motivatoren könnten prägende (negative) Ereignisse (2.6) oder aber die Partnerschaft sein (2.7). Finanzielle Anreize wie die direkte Kostenübernahme von Eintrittskosten wirken sich ebenfalls positiv auf die tatsächliche Umsetzung aus, argumentieren Befragte mit Verweis auf andere Formate wie das Funktionstraining der Rheumaliga (2.8). Zudem gehen ÄrztInnen davon aus, dass PatientInnen mit geringer körperlicher Belastung im Beruf die Empfehlung des RfB eher umsetzen als PatientInnen mit einer höheren körperlichen Belastung (2.9).

\section{Hemmnisse der PatientInnen, Bewegungsempfehlung} umzusetzen

Die Befragten nennen auch zahlreiche Gründe, warum Patientlnnen die Empfehlung des RfB nicht umsetzen. Dazu zählen die fehlende finanzielle Unterstützung der Bewegungsprogramme durch Krankenkassen (2.10) und lange Anfahrtswege zu angebotenen Bewegungsprogrammen (2.11). Neben niedriger "Compliance“ (2.12) sehen Befragte wiederholt Bequemlichkeit (2.13) und eine fehlende intrinsische Motivation (2.14) als Hemmnis bei der tatsächlichen Umsetzung. Schamgefühle hinsichtlich des eigenen Körpers oder der eigenen Unsportlichkeit könnten PatientInnen ebenfalls davon abhalten, ein RfB umzusetzen (2.15). Auch die fehlende Unterstützung durch das private Umfeld hat laut Befragten eine negative Wirkung (2.16). Hohe berufliche Belastungen werden von ÄrztInnen als ein zusätzlicher Faktor gesehen, der die Umsetzung eines RfB hemmen kann (2.17). 
Nachhaltigkeit der Bewegungsempfehlung

Befragte bemängeln immer wieder die fehlende Nachhaltigkeit, da PatientInnen nicht konsequent und anhaltend die Bewegungsempfehlungen umsetzen (2.18). Jedoch kann eine Reaktivierung früherer sportlicher Aktivitäten die Nachhaltigkeit der Bewegungsempfehlung verbessern (2.19). Laut Befragten leidet die nachhaltige Umsetzung unter einer fehlenden Kontrolle (2.20). Das RfB habe wiederum das Potenzial, die Nachhaltigkeit anderer Maßnahmen zu fördern, indem es an vorangegangen Bewegungsempfehlungen wie „Rehasport“ anknüpfe (2.21). Ärztlnnen sehen jedoch immer die Wahrscheinlichkeit, dass PatientInnen trotz einer schriftlichen Bewegungsempfehlung letztlich in erlernte Verhaltensmuster zurückfallen (2.22).

\section{Verbesserungsvorschläge der interviewten ÄrztInnen}

Verbesserungsvorschläge zielen $v$. a. auf die finanzielle Unterstützung der PatientInnen (3.1) und Ärztlnnen durch Krankenkassen $a b$ (3.2). Zudem wird vorgeschlagen, PatientInnen in allen Bereichen mit dem Thema Bewegung in Berührung zu bringen, indem es von verschiedenen ÄrztInnen beworben, aber auch medial stärker aufgegriffen wird (3.3).

\section{Diskussion}

\section{Methodik}

Die qualitative Erhebung erlaubte es, Haltungen und persönliche Einstellungen der ÄrztInnen zu dem RfB zu erheben. Jedoch sind die Ergebnisse auf Grund der zeitlichen Limitierung der Interviews auf 10 Minuten von eingeschränkter Tiefe. Trotz der geringen Fallzahl von acht Interviews zeichneten sich dennoch inhaltliche Wiederholungen im Antwortverhalten der Befragten ab und die letzten beiden ausgewerteten Interviews brachten keine wesentlichen thematischen Neuerungen. Entsprechend kann annäherungsweise von einer thematischen Sättigung im erhobenen Interviewmaterial ausgegangen werden [12]. Eine Ausdifferenzierung der Fragen und die Möglichkeit, im Interviewverlauf bei Passagen nachzuhaken, hätten aber mit hoher Wahrscheinlichkeit nochmals andere und vertiefende Perspektiven hervorgebracht. Die vorgestellten Ergebnisse sind daher als erste Themenübersicht zu verstehen. Zu bedenken ist auch, dass sich vermutlich am ehesten die ÄrztInnen zum Interview bereit erklärt haben, die eine eher positive Haltung zum RfB haben. Auch ist zu bedenken, dass es sich bei der Erhebung von patientenbezogenen Faktoren um Einschätzungen aus der Perspektive der ÄrztInnen handelt, nicht um die Perspektiven der PatientInnen selbst. Die Befragung von PatientInnen oder auch ÜbungsleiterInnen hätte gegebenenfalls nochmals andere Aspekte miteingebracht. Gleichwohl konnte durch die qualitative Befragung eine große thematische Bandbreite abgebildet werden. Diese beinhaltet nicht nur Hemmnisse bei der Ausstellung des RfB durch ÄrztInnen und Umsetzung der Bewegungsempfehlungen durch die PatientInnen, sondern dokumentiert auch förderliche Faktoren mit Blick auf die künftige Verwendung des RfB auBerhalb des Modellvorhabens.

\section{Ergebnisse zu den zentralen Fragestellungen}

Haltung

Die interviewten ÄrztInnen zeigten sich dem RfB gegenüber durchaus aufgeschlossen. Sie sahen es unter anderem als eine Möglichkeit, die im motivierenden Gespräch formulierten Empfehlungen zu gesundheitsförderlicher körperlicher Aktivität schriftlich zu fixieren und damit die Nachhaltigkeit zu verbessern. Auch stellte für sie das RfB eine Möglichkeit dar, um Bewegung an sich mehr Wertigkeit zu verleihen. Für die tatsächliche Umsetzung sahen sie neben ihrem eigenen Einfluss v. a. die soziale Kontrolle durch Dritte und die niedrigschwellige Einbindung von Bewegung in den Alltag als förderlich. Interessanterweise setzte sich kein/e InterviewpartnerIn damit auseinander, dass über das RfB auf besondere, mit dem Qualitätssiegel SPORT PRO GESUNDHEIT zertifizierte Bewegungsangebote verwiesen wird. Es muss an dieser Stelle offen bleiben, ob der Inhalt des Zertifikates den ÄrztInnen nicht bekannt war oder ob Bewegung allgemein, d. h. unabhängig von einer Zertifizierung, als gesundheitsförderlich empfunden wurde.

\section{Rahmenbedingungen}

Aus der qualitativen Befragung lässt sich schlussfolgern, dass für die Ausstellung des RfB nicht allein Rahmenbedingungen entscheidend sind. Eine ebenso zentrale Rolle nehmen auch die Hintergründe der PatientInnen (Adhärenz, Motivation, sportliche Vorerfahrungen, berufliche Belastungen) und v. a. deren Einschätzung durch die ÄrztInnen ein. So ist ein hemmender Faktor bei der Ausstellung die Frustration der ÄrztInnen über die schleppende Umsetzung der Bewegungsempfehlung bei den PatientInnen, deren Gründe die ÄrztInnen wiederum durchaus zu kennen glauben.

\section{Weitere hemmende Faktoren}

Einige ÄrztInnen gaben in der standardisierten Befragung durch die LÄK Baden-Württemberg an, das RfB nicht oder nur selten ausgestellt zu haben. In der Befragung durch die LÄK Baden-Württemberg wurden verschiedene Antwortkategorien für hemmende Faktoren der Ausstellung vorgegeben. Aus diesen nannten die befragten ÄrztInnen v. a. Rahmenbedingungen wie einen Mangel an Angeboten durch örtliche Sportvereine und fehlende (direkte) Kostenübernahmen durch die Krankenkassen. Durch die qualitative Befragung konnten jedoch noch weitere hemmende Faktoren ausdifferenziert werden. Diese lassen sich nach hemmenden Faktoren für die Ausstellung und deren Umsetzung aufgliedern.

Ein hemmender Faktor bei der Ausstellung stellt u. a. die Einschätzung der ÄrztInnen über die mangelnde Adhärenz der PatientInnen dar, d. h. die nur geringe oder nicht anhaltende Umsetzung der Bewegungsempfehlung. Die Frustration der ÄrztInnen über die fehlende Umsetzung von Empfehlungen durch die PatientInnen verstärkt die fehlende oder seltene Ausstellung des RfB. Auch ist manchen ÄrztInnen der Mehrwert des RfB nicht erkennbar, da sie ihre eigene Fähigkeit, die PatientInnen zu mehr Bewegung zu motivieren, als hoch genug oder die Wirkung des RfB als nicht nachdrücklich genug einschätzen. Weitere hemmende Faktoren sind fehlende Kenntnisse über das RfB und ein antizipiertes Überangebot an Möglichkeiten für die PatientInnen, in denen die Empfehlung des RfB untergeht.

Als hemmende Faktoren bei der Umsetzung nennen die Ärztlnnen nicht nur Rahmenbedingungen, sondern auch persönliche 
Merkmale der PatientInnen wie Motivation bzw. Bequemlichkeit, erlernte Verhaltensmuster, Schamgefühle, berufliche Belastung und das soziale Umfeld. Zusammenfassend liegen die hemmenden Faktoren, die in der qualitativen Befragung deutlich werden, v. a. auf der individuellen Ebene und im Bereich der Beziehung zwischen ÄrztInnen und PatientInnen.

Fragebogengestützte veröffentlichte Evaluationen des RfB in anderen Bundesländern (derzeit Hamburg, Thüringen, Hessen und Bayern) [15-19] zeigten, dass das RfB von ÄrztInnen, Sportvereinen und PatientInnen als sinnvoll angesehen wird. Dennoch gaben die ÄrztInnen auch hier unterschiedliche hemmende Faktoren sowohl für die Ausstellung als auch für die Umsetzung der Empfehlung durch die PatientInnen an. So wurden von den ÄrztInnen die fehlende Bereitschaft der Patienten, Bewegungsempfehlung umzusetzen, und die geringen Kenntnisse der Ärztlnnen über das RfB als hemmende Gründe für die Ausstellung angegeben. Als hemmender Faktor bei der Umsetzung wurde v. a. die fehlende Kostenübernahme durch die Krankenkassen erwähnt. Die von uns vorliegend herausgearbeiteten Erkenntnisse vertiefen oder erweitern die aus den genannten (teil-)standardisierten Befragungen gewonnenen Ergebnisse, was v. a. an dem vorliegend gewählten qualitativen Ansatz liegt. In den Interviews nennen die ÄrztInnen - neben dem möglichen Effekt des RfB - weitere Aspekte, die sie als förderlich bei der Umsetzung von Bewegungsempfehlungen sehen und die beim Verwenden des RfB zu beachten sind, z. B. die soziale Unterstützung. In der vorliegenden qualitativen Evaluation wie in den zitierten (teil-)standardisierten Befragungen werden Rahmenbedingungen als eher hemmend sowohl für die Ausstellung des RfB als auch die Umsetzung der Bewegungsempfehlung bewertet. Allerdings ermöglicht das in den Interviews gewonnene Material eine differenziertere Analyse der von den ÄrztInnen genannten hemmenden Faktoren. So sprechen die ÄrztInnen auch über ihr eigenes Empfinden bei der Beratung und reflektieren ihre eigene Haltung in Bezug auf die (antizipierte) Bereitschaft der PatientInnen, die Bewegungsempfehlung umzusetzen. Somit kann der in den zitierten Befragungen als hemmender Faktor genannte Aspekt „geringe Bereitschaft der Patienten zu sportl. Bewegung“ in der qualitativen Befragung deutlich differenzierter betrachtet werden.

Die dargestellten Ergebnisse unterstützen und ergänzen aber auch bereits vorangegangene Untersuchungen zu Umsetzungsproblemen der Gesundheitsberatung in der täglichen ärztlichen Begleitung von PatientInnen. So wurden bereits 2006 in einer Übersichtsarbeit für Deutschland fehlende Zeit, fehlende Vergütung und unzureichende ärztliche Qualifikationen als hemmende Faktoren für die Umsetzung dargestellt [20]. Dies resultiert auch aus internationalen Studien [21]. Eine aktuelle Analyse der gesundheitsökonomischen Wirksamkeit von bewegungsbezogenen Präventionsmaßnahmen zeigte, dass Überweisungsprogramme wie ein RfB, wenig Einfluss auf die Steigerung der Bewegung haben [22]. Dass finanzielle Anreize, wie z. B. ein Bonussystem der Krankenkassen, wenig Einfluss auf das Gesundheitsverhalten von PatientInnen haben, wurde bereits im Jahr 2005 empirisch im Gesundheitsmonitor der Bertelsmann Stiftung festgestellt [23] und im Jahr 2016 mit Bezug zur Nationalen Empfehlung zu Bewegung und Bewegungsförderung des Bundesgesundheitsministerium erneut formuliert [24].
Im Gegensatz zu diesen Erkenntnissen und Einschätzungen wurde von manchen der Befragten allerdings die fehlende direkte finanzielle Unterstützung der Bewegungsprogramme durch Krankenkassen als ein hemmender Grund für die Umsetzung von „mehr Bewegung “ durch die PatientInnen angegeben. Die indirekte finanzielle Unterstützung (d. h. Erstattung von Teilnehmergebühren auf Antrag) wurde in den Interviews nicht angesprochen. Bei den Aussagen der Befragten handelt es sich jedoch um Fremdeinschätzungen. In der o.g. Befragung 2005 zeigt sich, dass für die Steigerung der Bewegung intrinsische Motive der PatientInnen und die Sorge um die eigene Gesundheit ein viel entscheidender Faktor sind als eine finanzielle Unterstützung [23]. Diese zentrale Bedeutung der eigenen Motivation der PatientInnen wurde auch von einem der Befragten als ein Hemmnis der Umsetzung von Bewegungsempfehlung genannt.

\section{Ausblick}

Der Vergleich der Befragungsdaten aus den beiden deutschen Gesundheitssurveys GNHS-98 (Jahre 1997-1999) und DEGS 1 (20082011) weist darauf hin, dass ÄrztInnen im Zeitverlauf seltener allgemeine Beratungen zur Förderung körperlicher Aktivität, jedoch mehr individualmedizinisch ausgerichtete Beratungen zum krankheitsspezifischen Verhalten durchführen [25]. Zugleich war in den genannten Survey-Daten eine ärztliche Bewegungsempfehlung mit einer häufigeren Teilnahme an gesundheitsförderlichen Bewegungsangeboten assoziiert [25]. Diese positive Assoziation wird in der Selbstsicht von ÄrztInnen auf ihre Beratungskompetenz und ihre Fähigkeit, PatientInnen zur körperlichen Aktivität zu motivieren, allerdings nicht reflektiert, wie Befragungsdaten aus dem Zeitraum Oktober 2011 bis März 2012 zeigen [26]. Hier gab die deutliche Mehrheit (87\%) der an der Befragung teilnehmenden HausärztInnen ( $n=4074$ ) zwar eine hohe Beratungskompetenz an, schätzte zugleich jedoch die eigene Fähigkeit, Patientlnnen zu körperlicher Aktivität zu motivieren, mehrheitlich (52\%) als „nicht gut“ ein [26]. Im Gegensatz hierzu legt die umfangreiche Analyse einer PatientInnenbefragung zum RfB nahe, dass die RfB-bezogene Beratung zur Inanspruchnahme von Bewegungsangeboten motivieren kann - und dies auch bei Personen möglich ist, die eher schlecht für die Prävention angesprochen werden können [27]. Diese positive Haltung und Einschätzung aus PatientInnensicht könnte zukünftig vermehrt an ÄrztInnen vermittelt werden mit dem Ziel, die teilweise negative Einschätzung der ÄrztInnen zu patientenseitigen Faktoren zu verändern und damit die ärztliche Motivation zur Bewegungsempfehlung ggf. unter Verwendung des RfB oder Anwendung spezifischer Beratungstechniken zu erhöhen.

\section{Ethikvotum}

Für die vorliegend dargestellte qualitative Studie liegt ein positives Votum der Ethikkommission an der Medizinischen Fakultät und am Universitätsklinikum Tübingen vor (Nr. 511/2017BO2).

\section{Finanzierung}

Die vorliegende Studie wurde selbst initiiert und aus Eigenmitteln finanziert. Das Institut für Arbeitsmedizin, Sozialmedizin und Versorgungsforschung, Universitätsklinikum Tübingen, erhält eine in- 
stitutionelle Förderung durch den Verband der Metall- und Elektroindustrie Baden-Württemberg e.V. (Südwestmetall).

\section{Danksagung}

Die Autorinnen danken allen teilnehmenden ÄrztInnen und der Landesärztekammer Baden-Württemberg. Das Institut für Arbeitsmedizin, Sozialmedizin und Versorgungsforschung, Universitätsklinikum Tübingen, erhält eine institutionelle Förderung durch den Verband der Metall- und Elektroindustrie Baden-Württemberg e.V. (Südwestmetall).

\section{Interessenkonflikt}

Die Autorinnen/Autoren geben an, dass kein Interessenkonflikt besteht.

\section{Literatur}

[1] Foster C. Guidelines for health-enhancing physical activity promotion programmes. Oxford: Citeseer; 2000

[2] Lund K, Sibilitz KL, Berg SK et al. Physical activity increases survival after heart valve surgery. Heart 2016; 102: 1388-1395

[3] Brown JC, Winters-Stone K, Lee A et al. Cancer, physical activity, and exercise. Compr Physiol 2012; 2: 2775-2809

[4] Bundesärztekammer Rezept für Bewegung: Bundesärztekammer; [cited 2019 July $14^{\text {th }}$ ]. Available from https://www.bundesaerztekammer.de/aerzte/versorgung/praevention/sport-und-praevention/ rezept-fuer-bewegung/

[5] Württembergischer Landessportbund e.V Gibt es bestimmte Qualitätskriterien, die zu beachten sind? [cited 2019 July 2nd]. Available from https://www.wlsb.de/sportentwicklung-ehrenamtsportstaettenbau-schule-sportabzeichen/sport-ist-gesund/sport-progesundheit

[6] Anonym Rezept für Bewegung. Ärzteblatt Baden-Württemberg 2015; 70: 211

[7] Landesärztekammer B-W. Rezept für Bewegung 2015 [cited 2019 January $21^{\text {st }}$. Available from https://www.aerztekammer-bw.de/ news/2015/2015-04/rezept-fuer-bewegung/

[8] Hauser R Dr. med. Dipl. Inform. Persönliche Mitteilung. Landesärztekammer Baden-Württemberg 23.11.2016

[9] Mayring P. Kombination und Integration qualitativer und quantitativer Analyse. Forum Qualitative Sozialforschung 2001; 2: 11

[10] Kleining G. Umriss zu einer Methodologie qualitativer Sozialforschung. Kölner Zeitschrift für Soziologie und Sozialpsychologie 1982; 34: 224-253

[11] Helfferich C. Die Qualität qualitativer Daten. Manual für die Durchführung qualitativer Interviews. Wiesbaden: VS Verlag für Sozialwissenschaften. 2011
[12] Kruse J. Qualitative Interviewforschung: Ein integrativer Ansatz. Weinheim: Beltz Juventa. 2014

[13] Mayring P. Qualitative Inhaltsanalyse: Grundlagen und Techniken. Weinheim. Beltz; Verlag: 2003

[14] Emerich S, Preiser C, Rieger M. Begleitevaluation des „Rezept für Bewegung“ im Bereich der Landesärztekammer Baden-Württemberg. Unveröffentlichter Abschlussbericht zur Vorlage bei der Landesärztekammer Baden-Württemberg 17.12.2017

[15] Hübscher J. Evaluation der Umsetzung des „Rezeptes für Bewegung“ in Thüringen. Ärzteblatt Thüringen 2016; 27: 159-161

[16] Pfeiffer I, Kunze F. Kurzzusammenfassung der Evaluation des Rezepts für Bewegung in Hamburg.2016 [cited 2019 July $6^{\text {th }}$ ]. Available from https://www.hamburger-sportbund.de/system/files/downloads/files/ zusammenfassung_der_befragung_rezept_fuer_bewegung_endversion. pdf

[17] Curbach J, Apfelbacher C, Schemm A et al. Implementierung des „Rezepts für Bewegung“ durch niedergelassene Ärzte - Ausgewählte Ergebnisse einer standardisierten Befragung in Bayern. Das Gesundheitswesen 2014; 76: 08/09

[18] Gubisch R, Hoppe I, Vogt L et al. Wirksamkeit des Rezepts für Bewegung aus Patientensicht: ärztl. Beratung und Handlungskonsequenzen. Dtsch Z Sportmed 2014; 65: 206

[19] Loss J, Sauter A, Curbach J. Das Rezept für Bewegung als Maßnahme ärztlicher Prävention. Public Health Forum 2018; 26: 120-122

[20] Henke R, Kunstmann W. Gesundheitsberatung als ärztliche Aufgabe. Prävention und Gesundheitsförderung 2006; 1: 115-120

[21] Hebert ET, Caughy MO, Shuval K. Primary care providers' perceptions of physical activity counselling in a clinical setting: a systematic review. Br J Sports Med 2012; 46: 625-631

[22] Rütten A, Abu-Omar K, Burlacu I et al. Sind Präventionsmaßnahmen zur Bewegungsförderung kosteneffektiv? Ein systematischer Review von Überblicksarbeiten. Das Gesundheitswesen 2017; 79: 51-59

[23] Kunstmann W. Steuerungsperspektiven für Anbieter und Nutzer. „In“ Böcken J, Braun B, Schnee M, Amhof R editors Gesundheitsmonitor 2005 Die ambulante Versorgung aus Sicht von Bevölkerung und Ärzteschaft. Gütersloh: Bertelsmann Stiftung; 2005: 54-66

[24] Rütten A, Pfeifer K. Nationale Empfehlungen für Bewegung und Bewegungsförderung. Köln: Bundeszentrale für gesundheitliche Aufklärung 2016; [cited 2019 January 21 nd]. Available from https:// www.bundesgesundheitsministerium.de/fileadmin/Dateien/3_ Downloads/B/Bewegung/Nationale-Empfehlungen-fuer-Bewegungund-Bewegungsfoerderung-2016.pdf

[25] Gabrys L, Jordan S, Schlaud M. Prevalence and temporal trends of physical activity counselling in primary health care in Germany from 1997-1999 to 2008-2011. Int J Behav Nutr Phys Act. 2015; Oct 26 12: 136

[26] Diehl K, Mayer M, Mayer F et al. Physical activity counseling by primary care physicians: attitudes, knowledge, implementation, and perceived success. J Phys Act Health 2015; 12: 216-223

[27] Vogt L, Hoppe I, Thoma R et al. Wirksamkeit des Rezepts für Bewegung aus Patientensicht - die ärztliche Beratung und Handlungsabsichten/-konsequenzen nach erfolgter Rezeptverschreibung. Dtsch Med Wochenschr 2019; 144: e64-e69 